\title{
Anabases
}

ANABASES Traditions et réceptions de l'Antiquité

\section{La crisi dell'Impero romano come paradigma di quella europea : Ortega y Gasset}

\section{Arnaldo Marcone}

\section{(2) OpenEdition}

1 Journals

Edizione digitale

URL: http://journals.openedition.org/anabases/1600

DOI: 10.4000/anabases. 1600

ISSN: 2256-9421

\section{Editore}

E.R.A.S.M.E.

\section{Edizione cartacea}

Data di pubblicazione: 1 ottobre 2005

Paginazione: 101-112

ISSN: 1774-4296

\section{Notizia bibliografica digitale}

Arnaldo Marcone, « La crisi dell'Impero romano come paradigma di quella europea : Ortega y Gasset

», Anabases [Online], 2 | 2005, Messo online il 01 juillet 2011, consultato il 14 novembre 2019. URL: http://journals.openedition.org/anabases/1600 ; DOI : 10.4000/anabases. 1600 
Anabases 2 (2005), p. 101-112

\section{La crisi dell'Impero romano come paradigma di quella europea : Ortega y Gasset 1}

ARNALDO MARCONE

Qui avete l'origine della storia. L'uomo fa storia perché di fronte al futuro che non è nelle sue mani si trova che l'unica possibilità è il suo passato.

Ortega y Gasset

LA CADUTA DELL'IMPERO romano è notoriamente un paradigma classico nella cultura occidentale che si presta ad essere utilizzato come termine di confronto per fasi storiche percepite come di crisi o di decadenza dalla coscienza collettiva ${ }^{2}$. Nella recente storia europea fu soprattutto il primo conflitto mondiale ad essere vissuto, specialmente in Europa, come un evento drammatico, un punto di non ritorno che apriva scenari angoscianti.

L'idea di un declino, di un processo quasi ineluttabile di decadenza, di crisi dell'Occidente fece la fortuna del libro, destinato a diventare immediatamente un best-

1 E' notoriamente difficile ricostruire con esattezza la data della pubblicazione originaria degli scritti di Ortega. I riferimenti che si danno qui devono dunque intendersi attendibili ma non assolutamente esatti. Scrive lo stesso Ortega (La ribellione delle masse = RM, trad. it. SE, Milano, 2001, p. 56 n. 2) : "A quegli stranieri che generosamente scrivono sui miei libri e che talvolta incontrano difficoltà nel precisare la data della loro prima apparizione, desidero qui ricordare che la quasi totalità della mia opera ha visto la luce nella forma di articoli di giornali ; inoltre una sua gran parte ha tardato numerosi anni prima di trasformarsi in libro. "

2 Cf. A. DemandT, Der Untergang Roms als Menetekel ora in ID., Geschichte der Geschichte. Wissenschaftshistorische Essays, Köln-Weimar-Wien, 1997, p. 39-59. 
seller, di Oswald Spengler, Der Untergang des Abendlandes, il cui primo volume apparve nel $1918^{3}$. Il destino, davvero singolare, del libro, che richiamava nel titolo quello di un'opera da poco pubblicata da O. Seeck sulla fine dell'Impero romano (la Geschichte des Untergangs der antiken Welt pubblicata a partire dal 1895) fu quello di rappresentare un termine di riferimento costante per il «travaglio spirituale » (sono parole di Santo Mazzarino) suscitato da quel conflitto.

Proprio rispetto a tale travaglio, intellettuale oltre che spirituale, Ortega y Gasset occupa un posto particolare ${ }^{4}$. Con poche eccezioni, tra le quali senz'altro si deve segnalare quella di Santo Mazzarino, la riflessione dello scrittore spagnolo sul mondo antico e sulla sua crisi è stata in genere sottovalutata ${ }^{5}$. Pur originale e penetrante, caratterizzata da un'innegabile concezione aristocratica delle relazioni e delle dinamiche sociali ${ }^{6}$, essa può apparire a prima vista meno compiuta e organizzata di quella di altri pensatori contemporanei.

E' opportuno ricordare, in proposito, come il pensiero di Ortega si sia espresso per lo più nella forma di interventi giornalistici, di brevi scritti occasionali, di elzeviri che sono alla base anche delle opere più note. Si spiega così anche un aspetto della sua produzione che colpisce subito chi percorra anche rapidamente la raccolta dei suoi scritti : spesso si tratta di risposte, commenti o reazioni a opere di altri studiosi (Spengler e Toynbee in primo luogo) da Ortega letti in controluce rispetto alle proprie prese di posizione. Da un'eleborazione intellettuale di tal genere scaturisce inevitabilmente un aspetto di una certa occasionalità della sua produzione che trova una ricom-

3 Fondamentali sulla prospettiva in cui Spengler concepì il suo libro (di cui si deve ricordare il sottotitolo esplicativo : Umrisse einer Morphologie der Weltgeschichte) sono le osservazioni di A. DEMANDT, Spengler und die Spätantike (ora in ID., Geschichte, p. 60-80).

4 Le Obras Completas di Ortega sono pubblicate in 11 volumi, sotto la direzione di P. Garagorri, per la Casa Editrice "Revista de Occidente » di Madrid (un dodicesimo volume è destinato all'epistolario e ai corsi universitari). La bibliografia di e su Ortega è molto vasta. Un'informata e utile introduzione al suo pensiero politico e sociale si può leggere in J. Ortega y Gasset, Scritti politici (a cura di L. Pellicani e A. Cavicchia SCALAMONTI), Utet, Torino, 1979, p. 9-105.

5 S. MAZZARINO fa più volte riferimento alle tesi di Ortega in La fine del mondo antico, Milano, 1959 (2 ed. 1998). Si deve per altro osservare che la discussione che Mazzarino fa di La ribellione delle masse risente di un'erronea citazione di un passo del libro. Si veda p. 173 (cito dalla riedizione del 1998) : "La storia dell'impero romano è altresì la storia del suo sovvertimento, dell' impiego (corsivo mio) delle masse che assorbono e annullano le minoranze dirigenti e si sostituiscono ad esse. " In realtà Ortega ha scritto che "la historia del Imperio romano es la historia... del imperio de las masas" (dunque non è questione di " impiego delle masse »).

6 Giuste in proposito le osservazioni di MAZZARINO, lafine. Mi sembra invece che sia troppo propenso a valorizzare la componente liberale del pensiero di Ortega e a minizzare quella aristocratica, elitaria per la quale è evidente l'influenza di Nietzsche L. PELLICANI nella sua peraltro informata introduzione a La ribellione delle masse (Bologna, Il Mulino, 1984) p. 7-24. 
posizione e sistemazione nel quadro complessivo dell'opera. Anche il carattere della riflessione di Ortega sulla decadenza dell'Impero romano ha un carattere simile. Essa è tutt'altro che sistematica ma appare frutto di originali riflessioni all'interno di problematiche di ordine più generale.

Si tenga presente che Ortega aveva già avuto modo di confrontarsi con la storia di Roma in una delle sue opere più significative, Spagna invertebrata, la cui prima edizione apparve a Madrid nel 1923. Il libro si apre infatti con un appassionato riconoscimento del valore esemplare della storia romana : « Il popolo romano costituisce un caso unico nel complesso delle conoscenze storiche : è l'unico popolo che sviluppa per intero il ciclo della sua vita davanti alla nostra contemplazione. Possiamo assistere alla sua nascita e alla sua estinzione [...]. Roma è, dunque, l'unica traiettoria completa di un organismo nazionale che conosciamo ${ }^{7}$."

Non diversamente da Spengler Ortega dimostra una sensibilità peculiare per la ricerca di costanti nel divenire storico. Tale ricerca lo avvicina anche alla riflessione di Arnold Toynbee con la cui opera ebbe un controverso e intenso confronto intellettuale ${ }^{8}$. Sociologo, storico se non vero e proprio filosofo della storia Ortega si confrontò con il problema della decadenza di Roma in modo del tutto originale. Interessato alla fase creativa della civiltà romana, ammiratore di Mommsen e, attraverso di lui, di Cesare sembra essersi accostato al problema della decadenza di Roma fondamentalmente attraverso la Social and Economic History of the Roman Empire di M. Rostovtzeff, pubblicata a Oxford nel 1926 ?.

Nella ricostruzione rostovtzeffiana a colpire i contemporanei furono subito alcuni aspetti che suscitarono reazioni discordanti. In primo luogo era evidente che lo storico russo era fortemente influenzato dalla storia contemporanea, soprattutto daquella del suo paese di origine, la Russia, e che faceva ricorso con disinvoltura a concetti come " borghesia ", " capitalismo ", " proletariato " e così via ${ }^{10}$. Essi presupponevano un'idea fondamentalmente modernizzante dell'economia antica che era ed è tutt'altro che pacifica ${ }^{11}$. Ma a colpire era soprattutto la sua interpretazione della fine del mondo, attribuita a una fatale coalizione realizzatasi tra i contadini poveri e i soldati nel III secolo

7 P. 521-522 dell' edizione degli scritti politici di Ortega citata sopra.

8 Mi riservo di dar conto in un prossimo contributo della serrata discussione che Ortega riserva a A Study of History di A. Toynbee in Una interpretación de la historia universal, Madrid, 1960 (trad. it., Milano, 1978).

$9 \quad$ La cosa è ben vista da S. Mazzarino.

10 La rivisitazione della crisi del III secolo in chiave di storia contemporanea era peraltro comune a molti storici attivi subito dopo la fine della Prima Guerra Mondiale. In proposito ha ben scritto S. Mazzarino : " Poco a poco, il crepuscolo di Roma finì con l'apparire, a molti degli storici fra il 1920 e il 1930, quasi una pagina di storia contemporanea " (lafine, p. 178).

11 Tale modernizzazione dell'economia antica ha trovato un severo censore soprattutto in Moses Finley seguito da molti suoi allievi. 
contro la civiltà urbana ${ }^{12}$. Fu facile per i critici vedere in questa tesi la trasposizione della situazione che aveva portato in Russia alla rivoluzione sovietica del 191713.

Non c'è dubbio che Rostovtzeff fosse emotivamente coinvolto dalla fragilità della borghesia del suo paese che non era stata in grado di resistere alla rivolta delle masse «bramose di livellamento generale ». Il celebre interrogativo con cui si chiude la History, con il quale sembra escludersi la possibilità che una civiltà elevata possa essere assorbita dalle " masse " senza degradarsi, in verità, riflette preoccupazioni che, dopo la tragedia del primo conflitto mondiale, erano generalmente diffuse nella cultura occidentale : Spengler e Ortega y Gasset, per fare solo i nomi più famosi, si erano posti, in quei medesimi anni, analoghi interrogativi ${ }^{14}$.

Ortega registrò prontamente l'interrogativo di Rostovtzeff in uno dei suoi Fogli di Almanacco (una sorta di appunti occasionali dal contenuto libero) del 1926. Si tratta di un'annotazione molto partecipata.

"Nel 1926 Rostovtzeff pubblico il suo libro The social and economic History of the Roman Empire. E' il primo studio in grande che si fa dell'Impero romano e completa la ricostruzione della Repubblica genialmente tracciata da Mommsen ${ }^{15}$. "

Quest'annotazione è significativa a prescindere dalla correttezza della valutazione storiografica perché certo la History rostovtzeffiana non può essere considerata un " completamento » della storia della Repubblica romana di Mommsen. Né, malgrado il pessimismo dell'interrogativo finale, contrariamente a quello che talvolta si è sostenuto, il tono di fondo della History non è quello della sua conclusione. Si deve essere chiari. In Rostovtzeff l'ansia da declino, da crisi di civiltà, che è così evidente nella storiografia del primo dopoguerra, si manifesta in un modo peculiare. La History è, in realtà, una brillante ricostruzione del successo di una civiltà garantito dall'affermarsi di un ceto che può essere definito, a buon diritto, "borghese ". Il suo crollo, interpretato in modo sommario, sulla base di un'analogia con le vicende della Rivoluzione d'Ottobre - come gli fu prontamente e aspramanente contestato - come dovuto a una

12 La tesi è in qualche modo anticipata alla fine del capitolo IV ove si parla di una crescente ruralizzazione nel reclutamento delle legioni soprattutto a partire dal regno di Marco Aurelio.

13 Particolarmente severa fu la recensione di H. LAST apparsa sul Journal of Roman Studies 16 (1926), p. 120-128. Last, che più tardi sarebbe diventato titolare della Camden Chair di Storia Antica a Oxford, riconosceva, peraltro, che le caratteristiche della History erano tali da farne un " classico " della storiografia moderna sull'Impero romano. La critica meglio documentata all'opera di Rostovtzeff resta quella di Meyer ReINHOLD, Historian of the Classic World : a critique of Rostovtzeff, Science and Society, 10 (1946), p. 361-391= ID., Studies in Classical History and Society, Oxford, 2002, p. 82-100.

14 Si veda di in particolare la seconda parte di Spagna invertebrata.

15 Cito da J. OrTega y Gasset, Lo Spettatore, a cura di C. Bo, Guanda, Milano, 1984, p. 203. 
saldatura tra contadini e soldati contro gli abitanti delle città, ha uno spazio relativamente minore nell'opera.

Fu senza dubbio la delicata temperie culturale del primo dopoguerra a far sì che fossero proprio le pagine dedicate da Rostovtzeff alla crisi del III secolo a meritare un'attenzione eccezionale. L'interrogativo risultava in effetti in sintonia con quello di tutta una generazione di studiosi. Le parole " terribili » di Rostovtzeff erano destinate a rimanere impresse. Con quelle Ortega si trovava in ovvia sintonia : "L'evoluzione del mondo antico contiene una lezione e un avvertimento anche per noi. La nostra civiltà non può continuare se non arriva ad essere una civiltà non di una classe ma delle masse. " Poco importa se la considerazione successiva appare francamente poco probabile : "Quelle orientali [le civiltà] furono più stabili e durature di quella grecoromana perché basandosi principalmente sulla religione erano più vicine alle masse. " Certamente è la parte finale del ragionamento di Rostovtzeff che doveva apparire fondamentale : "Un'altra lezione è che il tentativo di livellamento con mezzi violenti non è mai servito per elevare le masse. Queste hanno distrutto le classi superiori e sono riuscite solo ad accelerare il processo di imbarbarimento. Ma il problema ultimo rimane come uno spettro sempre presente e ineliminabile. E' possibile estendere una civiltà superiore alle classi inferiori senza abbasssare il loro livello e diluire fino ad annullarne la qualità ? Ogni civiltà non è condannata a decadere appena penetra nelle masse 16 ?"

Si deve osservare che questa riflessione di Rostovtzeff, così apprezzata per il riferimento a temi all'epoca vivacemente di attualità nel dibattito politico-sociale, ha un fondamento storico assai problematico. Le " masse " in quanto tali, infatti, hanno un ruolo assai difficilmente distinguibile nelle relazioni sociali dell'Impero romano. Più plausibile sarebbe stato, se mai, interrogarsi sul declino creativo della civiltà antica che avrebbe avuto poi un suo peculiare - e contraddittorio - prolungamento nel periodo storico che, in particolare a partire dal secondo dopoguerra, siamo soliti chiamare " Tarda Antichità 17 ". Rostovtzeff nel chiudere il libro con un interrogativo così vibrante di attualità sembra inclinare a sua volta verso temi di psicologia sociale, che

16 Rostovtzeff conclude con questa riflessione il capitolo XII della History (Il dispotismo orientale e la fine della civiltà antica). Per la risposta di Rostovtzeff alle sue critiche si veda, oltre alla nuova edizione della sua opera da me curata (che tiene conto di correzioni e di integrazioni di suo pugno in vista di una riedizione che non vide mai la luce), pubblicata da Sansoni (Milano, 2003), p. 740-742, il mio "Un inedito di Rostovtzeff sulle cause della caduta dell'Impero romano", Historia 48 (1999), p. 254-256.

17 Per il dibattito in corso sulla Tarda Antichità e la sua periodizzazione rimando ai contributi raccolti in Studi Storici 45 (2004), p. 5-46. 
pure gli erano poco congeniali, ma che erano evidentemente a loro volta influenzati da argomenti dibattuti negli Stati Uniti in cui era da poco arrivato dall'Inghilterra ${ }^{18}$.

L'attenzione che Ortega dimostra per la crisi dell'Impero romano ha un riscontro in un articolo apparso nel 1926, indicativo sin dal titolo : Sopra la morte di Roma ${ }^{19}$. L'occasione gli è fornita dalla traduzione spagnola appena pubblicata sulla "Revista de Occidente" (sul numero 37) del famoso saggio di Max Weber Die sozialen Gründe des Untergangs der antiken Kultur ${ }^{20}$. Il fatto che il saggio di Weber - a sua volta una rielaborazione di una conferenza di taglio semidivulgativo - preceda cronologicamente il libro di Spengler crea invero qualche imbarazzo a Ortega (fu pubblicato nel 1896), che infatti prescinde dalle radicali differenze che separano l'approccio weberiano da quello di Spengler 21.

In realtà è fondamentalmente il Kulturpessimismus di quest'ultimo che preme al pensatore spagnolo. A favore di Ortega gioca il titolo dello studio di Weber ove si parla di "Kultur », un termine dalle forti connotazioni spengleriane e che può creare qualche equivoco ${ }^{22}$. Ortega apre il suo articolo con delle considerazioni che appaiono in linea con lo spirito del tempo ma che sono in realtà diverse dai presupposti della riflessione di Weber : "La società in cui viviamo è il nostro suolo, il nostro spazio morale e ci sembra che morendo ci resti in qualche modo il nostro vuoto. L'idea che anche questo vuoto scompaia con tutto il corpo sociale che ci ha concordato e protetto dà l'ultimo colpo alla nostra morte e ci fa morire del tutto."

E' significativo come Ortega recepisca il significato della scossa impressa nelle coscienze europee dal primo conflitto mondiale. La considerazione che si riporta appare particolarmente lucida "Si ricordi la certezza con cui l'europeo di vent'anni fa dava per definitiva la forma europea del mondo. Si pensava che ormai fosse impossibile una cesura storica come quella atroce a cui soccombette l'Impero romano. E, ciò nono-

18 Precursore di questo tipo di ricerche si deve considerare il libro di G. LE Bon, Psychologie des Foules, Paris, 1895, che ebbe uno straordinario successo. Nel 1920 era già giunto alla sua ventesima edizione nel 1920 (l'ultima ultima edizione italiana, con prefazione di P. Melograni è apparsa da TEA a Milano nel 2004). In questa sede basterà aggiungere che nel 1923 S. Freud pubblicò a Leipzig il suo Massenpsychologie und Ich-Analyse.

19 Trad. it. in Lo spettatore, p. 132-141.

20 "Die Wahrheit ", Frommans Verlag, maggio 1896. Si veda la traduzione italiana in M. Weber, Storia economica e sociale dell'Antichità, Editori Riuniti, Roma, 1981, p. 371393. Il valore di questo saggio di Weber è stato invero in genere sopravvalutato.

21 Il libro di Spengler era stato tradotto in spagnolo a Madrid nel 1923 per la «Biblioteca de Ideas del Siglo XX ». Ortega si occupò direttamente del libro nel suo Prólogo a "La decadencia de Occidente " de Oswald Spengler che si può leggere in ID., Las Atlándidas y del Imperio Romano, Madrid, 19605, p. 155-159. Ringrazio Filippo Carlà per avermi procurato questo libro.

22 Si vedano le osservazioni di B. SPAGNUOLO VigORITA nell' " Avvertenza " alla citata traduzione italiana (p. XXIV). 
stante, i fatti dell'ultimo decennio hanno fatto vacillare questa convinzione. L'Europa sente che il suo polso si allenta e intravvede per la prima volta il pericolo di morte. Per questo è sorto dappertutto spontaneamente il tema delle decadenze 23 ".

Come si può ben capire Ortega è in sintonia con Weber nel ritenere che l'Impero romano sia caduto per cause interne. Weber aveva molto insistito su questo punto. Il suo saggio si apre infatti con una affermazione che non lascia adito a dubbi : « L'Impero romano non fu distrutto da forze estrinseche, come la superiorità numerica dei suoi avversari o l'incapacità dei suoi capi politici ${ }^{24}$. "

Ortega concorda perché, a suo modo di vedere, « i mondi (Ortega chiama Roma "un mondo") muoiono solo di morte naturale ». Attribuire la causa della fine dell'Impero di Roma alle invasioni dei barbari non è altro, che un' "invenzione " dei letterati della decadenza romana " che erano, come sogliono essere i letterati in tutte le epoche difficili, superlativamente reazionari. Incapaci a creare cultura, chiamavano così la tradizione 25 ".

In realtà il ragionamento di Ortega prescinde fondamentalmente dall'argomentazione di Weber e si muove lungo le linee che gli sono familiari. La sua convinzione di fondo è che Roma abbia pagato il fatto di non aver saputo adeguare il proprio sistema elettorale, proprio di una "democrazia seppure aristocratica ", alle nuove esigenze di uno Stato che si era allargato a comprendere tutta l'Italia. Concedere i pieni diritti politici agli italici non è che una concessione priva di contenuto perché questi non sarebbero mai potuti venire a Roma per votare. Un difetto « di tecnica elettorale » è dunque alla base della rovina dello Stato romano che non seppe escogitare una soluzione alla dissociazione tra la provincia e Roma (questo concetto è ripreso e sviluppato in "Rivoluzione delle masse"). E questo difetto si spiega sulla base dei limiti intrinseci allo spirito romano, molto dotato per il comando ma privo di agilità intellettuale.

Proprio per questo, a suo modo di vedere, predicare la tradizione è una stupidaggine perché sono le virtù che non abbiamo " quelle che importano di più ». Nella sua ammirazione per Cesare Ortega gli attribuisce l'intuizione della necessità che lo Stato dovesse mutare forma. A tal fine anziché indirizzare le sue conquiste verso le "caduche " province orientali si rivolse ai popoli giovani dell'Occidente. Lidea geniale che per salvare Roma bisognasse esaltare la provincia non poteva riuscire accettabile alle " teste putrefatte » della vecchia aristocrazia romana e men che mai al suo erede Augusto.

Ortega trae da questa sua peculiare interpretazione della storia della crisi dell'Impero romano una lezione non meno personale per il destino della Spagna e dell'Europa. Merita di riportare integralmente le sue parole :

\footnotetext{
23 P. 132-33 dell'ed. italiana citata prima.

24 Ortega (p. 136) dice di non comprendere perché Weber consideri una causa estrinseca l'incapacità dei capi politici.

25

P. 135 della trad. it.
} 
"In grande o in piccolo, ogni storia nazionale arriva a un punto in cui, per ricrescere, deve lasciare riposare il vecchio capitale e riporre tutte le speranze nella provincia : un momento in cui è necessario svegliare la periferia del grande corpo politico e gridare : Ehi voi province : bisogna che smettiate di essere provinciali! E' arrivato il momento che dovete avere pronti i vostri impulsi intatti. Lo Stato rinascerà da voi o non rinascerà. Ehi, province : in piedi 26 !"

Proprio le "masse ", in altre parole il nuovo contesto sociale che si stava realizzando in Occidente, il cui protagonista, l'uomo-massa appunto, è in realtà un primitivo che è inconsapevole del carattere artificiale della civiltà di cui gode i frutti, erano, come si è visto, al centro della riflessione di Ortega di quegli anni. Il primo capitolo del suo libro forse più famoso, "La ribellione delle masse", apparve nel 1927 sul quotidiano "Il Sol" con il titolo "Masas" 27.

La citazione di qualche passo può servire a dare un'idea della peculiarità della problematica che si deve presupporre alla base dell'opera :

Scrive Ortega : "Questo è il fenomeno formidabile del nostro tempo, prospettato senza dissimulare la brutalità della sua apparenza. E' inoltre di un'assoluta novità nella storia della nostra civiltà. Mai, in tutto il suo sviluppo, è accaduto nulla di simile. Se vogliamo trovare qualcosa di analogo, dobbiamo uscire dalla nostra storia e immergerci in un mondo, in un elemento vitale, completamente diverso dal nostro, dobbiamo penetrare nel mondo antico e spingerci fino all'ora del suo declino. La storia dell'Impero romano è anche la storia del sovvertimento e dell'imperio delle masse che assorbono e annullano le minoranze dirigenti e si sotituiscono a esse. Allora si produce anche il fenomeno dell'agglomeramento, del pieno. Per questo, come ha osservato assai bene Oswald Spengler, fu necessario costruire, proprio come ai giorni nostri, enormi edifici. L'epoca delle masse è l'epoca del colossale ${ }^{28}$."

In un capitolo successivo Ortega sostiene che la crisi dell'Occidente è dovuta al fatto che l'uomo medio, l'uomo-massa non riesce a stare al passo con la sua stessa civiltà che fondalmente non comprende ${ }^{29}$.

"Non credo che questo sia mai accaduto in passato. Tutte le civiltà si sono estinte per l'insufficienza dei loro principi. L'Europa minaccia di soccombere per il motivo opposto (sott. mia). In Grecia e a Roma non rovinò l'uomo ma i suoi principi, l'Impero romano decadde

P. 141 della trad. it.

Per il valore specifico del libro rispetto alla crisi del mondo antico si veda J. FERnándeZ UBINÁ, "La rebelión de las masas y el fin de la cultura clásica", in Actas del II Congreso Andaluz de Estudios Clasicos (vol. I), Antequera-Malaga 24-26 de Mayo 1984, O. GarCíA DE LA Fuente (ed.), Sociedad Española de Estudios Clásicos-Delegación de Málaga, Málaga, 1988, p. 133-139.

RM, p. 55.

RM, p. 116-117. 
per mancanza di tecnica ${ }^{30}$. Giunto a uno sviluppo demografico così alto, ed esigendo una così vasta comunità la soluzione di certe urgenze materiali che soltanto la tecnica poteva trovare, il mondo antico incominciò a subire un processo d'involuzione, a retrocedere e a consumarsi ${ }^{31}$. "

Si deve ricordare come Ortega si sia mostrato particolarmente ricettivo anche del nuovo orientamento di prospettiva culturale che gli studi etnologici, archeologici e preistorici stavano producendo all'inizio degli anni ' 20 (è notevole come sappia cogliere precocemente il declino delle discipline filologiche nel xx secolo) ${ }^{32}$. Una visita compiuta da Frobenius in Spagna non gli era passata inosservata 33.

Contro l'idea di monismo culturale per cui si parla di cultura geca, romana, orientale come se fossero stadi di un unico processo che le riassorbe, Ortega oppone l'acquisita consapevolezza che esistano sistemi culturali diversi dal nostro. " Pernicioso favoritismo " chiama l'idea tradizionale che vuole che Greci e Romani fossero i popoli classici, sostanziata dall'influenza che la nostra civiltà ha ricevuto da loro. Pur vicino alle idee di Frobenius e Spengler, accostate in verità forse con una certa libertà, Ortega non accetta di seguirli nel dar loro una " dimensione metafisica " : per essere feconde devono rimanere impiegate sul piano del puro empirismo storico ${ }^{34}$.

Si deve sottolineare ancora la particolare attenzione riservata da Ortega alle idee fortemente innovative in campo biologico sviluppate a partire dagli anni ' $20 \mathrm{da}$ J. von Uexküll ${ }^{35}$. Esse sono da lui avvicinate a quelle elaborate da Einstein nella fisica e da Spengler nella filosofia della storia 36 .

In sintesi la concezione della biologia che è alla base delle teorie di Uexküll si spiega come una risposta al materialismo di matrice darwinista che all'inizio del ' 900 faceva scuola (soprattutto nella versione che ne dava E. Haeckel) ${ }^{37}$. Contro il trionfo di derivazione fisico-chimica dei fenomeni naturali Uexküll è sostenitore di un "vitalismo " che presuppone l'accettazione di un principio immateriale nello sviluppo dei

30 In una rapida riflessione contenuta in un "Prólogo a dos ensayos de historiografía" di presentazione a L'autunno del Medio Evo di J. HuIZINGA e a La formazione del tipo culturale olandese di C. ANTONI (Revista de Occidente 145, luglio 1935 = Las Atlántidas, p. 161164) Ortega ribadisce : "Il mondo antico morì strangolato per la sua inadeguatezza nel domino tecnico della natura [...] La nostra cultura (civilización) corre un rischio analogo a quello che dissolse quella antica - analogo e opposto. Può morire per mancanza di tecniche morali. I nostri problemi non sono fisici ma di valori umani. "

31 RM, p. 117.

32 Etnología in Las Atlántidas, p. 25-27.

33 Si vedano "El Sentido historico" e "Las Ideas de León Frobenius" pubblicati sul giornale «El Sol » del 10 luglio 1924= Las Atlántidas, rispettivamente a p. 47-60 e 61-64.

34 Si veda il saggio "Los "ámbitos culturales" "in Las Atlántidas, p. 29-43.

35 Cf. G. Agamben, L'aperto, l'uomo e l'animale, Milano, 2002.

36 Prólogo a La decadencia d'Occidente de SPENGLER, p. 156.

37 Si veda l'introduzione di F. MONDELla a J. vON UEXKÜLl, Ambiente e comportamento, Milano, 1967. 
processi organici. Per capire perché le sue idee, in difesa di un'aristocrazia di valori morali, minacciata dalle idee materialistiche - in quanto tali svilenti la dimensione psichica dell'individuo - potessero essere in sintonia con quelle di Ortega basterà leggere questo passo :

"Se esistono solo forze fisiche e chimiche allora la conformità ad un piano che noi vediamo è solo apparenza. Allora il mondo che ci circonda, con le sue migliaia di forme e di colori, con tutte le sue disposizioni etiche ed estetiche svanisce in una danza di atomi, ove non regna altro che il numero. Al posto del crudele Jehova, che nel Medioevo reggeva il mondo, abbiamo posto un nuovo idolo, il numero. Se ne vedranno le conseguenze, solo che esso domini completamente le masse e le metta in movimento ${ }^{38}$."

A giudizio di Ortega la fondamentale intuizione di Uexküll e il progresso della sua teoria rispetto a quella di Darwin sta nel concepire un mondo animale che, anziché adattarsi all'ambiente (inteso come ambiente "biologico" che interagisce con l'organismo e non come puro dato fisico-chimico), opera in modo che sia quest ultimo ad adattarsi a lui. Una concezione di questo genere ha importanti conseguenze anche in campo etico e psicologico. Secondo Ortega, se si può banalizzare il suo pensiero, come è erroneo concepire la vita psichica come funzionale all'ambiente, come semplice ricettività e passività, così è sbagliata la pedagogia che svaluta e mortifica la vitalità insita nell'individuo.

Un saggio, dal titolo ingannevole, "Il don Chisciotte nella scuola 39", ha come senso profondo quello di « convincere la pedagogia a sottomettere tutta la prima fase dell'educazione all'imperativo della vitalità ». Perché preme tanto a Ortega la tutela del vitalismo innato nel bambino, perché la sua polemica contro il " metodo crudele di colpire l'infanzia e di produrre momini che portano dentro di sé un'infanzia incancrenita ? " La spiegazione risiede essenzialmente nell'idea di quello che Ortega dà del "paradosso del selvaggismo ". A suo modo di vedere sono le grandi esplosioni di "selvaggismo " a dar vita alla cultura e alla civiltà, alle grandi fasi di rinnovamento. Di qui il valore della vita primitiva considerata come " una fortuna inesauribile dell'organizzazione culturale e civile ». Contro l'uomo-massa che si compiace della sua presunta civiltà, Ortega perora quindi una pedagogia della creatività come premessa per un nuovo rapporto tra l'individuo e la società.

In realtà una delle idee più originali sviluppate da Ortega già a partire da Spagna invertebrata 40 riguarda proprio l'origine della società umana. A suo modo di vedere, contro le dottrine evoluzionistiche, essa ha solo somiglianze estrinseche con quelle animali. Né la si può considerare come uno sviluppo del gruppo familiare che, a rigore,

38 Cf. J. VON UEXKÜLL, Bausteine zu einer biologischen Weltanschauung, München, 1913, p. 263.

39 Si veda Lo Spettatore, p. 52-79, cit. a p. 67, p. 74.

40 La prima edizione di Spagna invertebrata fu pubblicata a Madrid nel 1923. 
fa la sua comparsa in un secondo tempo rispetto a quella che definiamo società ${ }^{41}$. Ortega osserva :

"Molteplici dati, soprattutto etnologici, obbligano a pensare che la società nasce dall'attrazione superiore che uno o diversi individui esercitano sopra gli altri. Le epoche di decadenza sono le epoche in cui la minoranza-guida di un popolo, l'aristocrazia, ha perduto le sue qualità supreme ${ }^{42}$. "

Nel cosiddetto "Prologo per i Francesi" alla Ribellione delle masse, che risale al 1937 43, con riferimento a un processo in cui il collettivo assorbe l'individuale, in cui la massa prevale sulla creatività del singolo, Ortega scrive :

"Così come vanno le cose, con la diminuzione progressiva della "varietà delle situazioni”, siamo incamminati per la via più breve verso il Basso Impero. Anche quella è stata un'epoca di massa e di spaventosa omogeneità. Già al tempo degli Antonini si avverte chiaramente uno strano fenomeno, meno sottolineato e meno analizzato di quanto si meriterebbe : gli uomini sono diventati stupidi. Il processo risaliva a tempo addietro. E' stato detto, con qualche ragione, che lo stoico Posidonio, maestro di Cicerone, è l'ultimo uomo antico capace di porsi di fronte ai fatti con la mente permeabile, attiva, disposta a interrogarli. Dopo di lui, le menti si chiudono e, salvo gli Alessandrini, non fanno altro che ripetere, riprodurre stereotipi ${ }^{44}$."

La riflessione di Ortega sull'Impero romano e sulla sua crisi è ripresa e organizzata in modo sistematico in un saggio specifico, Del Imperio Romano. Historia ascendente, pubblicato in forma di articoli su "La Nación" di Buenos Aires nel 1940. In realtà questa riflessione va oltre il problema della decadenza e contiene uno sguardo di prospettiva che merita di essere apprezzato ${ }^{45}$. Il saggio si apre con un premessa che contiene un significativo riconoscimento all'originalità della Storia economica e sociale dell'Impero romano di Rostovtzeff che avrebbe avuto il coraggio di riprendere e completare la storia romana al punto in cui era stata lasciata in sospeso da Mommsen, vale a dire alla morte di Giulio Cesare. L'importanza della storia romana si spiega, a giudizio di Ortega, perché questa « è paradigma di tutte le altre, per la sua materia e per il grado di maturità a cui è giunta la sua investigazione ». L'Impero romano è molto probabilmente « la realtà di maggiore tras-

41 Gli argomenti fondamentali sono riassunti nel saggio del 1924 "Non essere un uomo esemplare" (p. 97-101 della citata edizione a cura di C. Bo).

$42 \quad$ P. 97

43 Il "Prologo per i francesi" è la premessa scritta da Ortega nel 1937 per la traduzione francese di La ribellione delle masse. Le edizioni del libro successive a questa data includono anche il cosiddetto "Epilogo per gli inglesi", pubblicato l'anno dopo.

44 RM, p. 29-30 della trad. it.

45 Cito dall'edizione degli Scritti politici curata da L. PelliCani, p. 983-1025. La prima traduzione italiana di questo scritto figura in J. ORTEGA y GASSET, Schema delle crisi, trad. it., Bompiani, Milano, 1946, p. 125-169. 
cendenza finora manifesta nella storia umana " e questo rende comprensibile che un'opera come quella di Rostovtzeff abbia suscitato tanto interesse e tanti dibattiti. Il libro di Rostovtzeff è importante proprio perché ispirato dall'attualità. Mommsen, " una specie di Ercole della storia " si era fermato sulla soglia dell'Impero perché all'interesse per Roma repubblicana lo portavano le sue esperienze personali e quelle del suo tempo : "Il secolo XIX poteva comprendere soltanto la Roma repubblicana, la Roma ascendente. "

Il dato importante che emerge da questo saggio è che per Ortega, come in parte si è già visto, non esiste in realtà il problema della decadenza dell'Impero romano in quanto tale. L'Impero è, a suo modo di vedere, nel suo stesso costituirsi un prodotto di un processo di decadenza, l'esito necessario della crisi della Repubblica romana, della sua incapacità di aprirsi alle province e di valorizzarne il contributo innovativo e costruttivo. Dunque Ortega è lontano dalla prospettiva di Rostovtzeff per il quale la crisi (che è un fenomeno politico e sociale) intervenne nel III secolo dopo un periodo molto positivo, culminato nell'età degli Antonini. In realtà Ortega guarda lontano e cerca di trovare nel " paradigma » rappresentato dall'Impero Romano un messaggio da utilizzare in positivo per il futuro della storia europea 46 . Si deve riconoscere come l'attualizzazione che è alla base della sua interpretazione dell'Impero romano conservi intatto il suo fascino.

\begin{tabular}{l} 
Arnaldo MARCONE* \\
\hline Università di Udine \\
Dipartimento di Storia e \\
Tutela dei Beni Culturali \\
Via Petracco, 8 \\
I-33100 Udine (Italie) \\
arnaldo.marcone@uniud.it
\end{tabular}

46 Si veda almeno la conferenza "De Europa meditatio quaedam" letta a Berlino il 7 settembre 1949 e pubblicata postuma con il titolo "Meditazione sull'Europa" (p. 10291102 dell'edizione citata) in cui si riprendono alcune riflessioni sul destino del mondo antico come premessa per una considerazione della presente relatà europea. Merita di citare questo passo : "Nella Politica di Aristotele non c'è una visione di fatti politici macroscopici come le grandi monarchie macedone e persiana. E' sempre la polis, monarchica o no, che è davanti al suo sguardo [...]. Sarebbe ricadere nell'antica miopia non scoprire unità di potere pubblico se non dove questo ha assunto sembianze ormai note e come solidificate di Stato, cioè nelle nazioni europee [...]. L'unità dell'Europa non è una fantasia, ma è la realtà stessa e la fantasia è precisamente il contrario : credere che la Francia, la Germania, l'Italia o la Spagna sono realtà sostantive, pertanto, complete e indipendenti » (p. 1082-1083 Pelicani). Cf. F. Moiso- M. Cipolloni- J.C. LeVÊQue (éds), Atti del Convegno, Ortega y Gasset pensatore e narratore dell'Europa, (Milano-Gargnano del Garda 13-18 novembre 1998), Milano, 2001.

* Ringrazio l'amico Pablo Diaz per alcuni utili suggerimenti. 